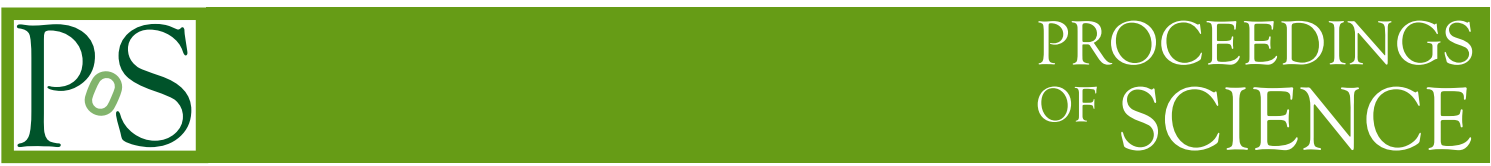

\title{
Recent W/Z results from CMS
}

\section{Rajdeep M Chatterjee ${ }^{* \dagger}$}

Tata Institute of Fundamental Research, Mumbai

E-mail: rmchatterjeejregmail.com

The production of $\mathrm{W}$ and $\mathrm{Z}$ bosons is studied in pp collisions at center-of-mass energies of both 7 and $8 \mathrm{TeV}$ using data collected by the CMS experiment. W events are characterized by an isolated, energetic electron or muon. $\mathrm{Z}$ events are characterized by a pair of isolated, energetic electrons or muons. Data-driven methods are used to estimate reconstruction and triggering efficiencies, as well as the yields of the main backgrounds. Recent results on $\mathrm{Z}$ boson differential cross section, $\mathrm{Z}$ boson angular coefficients, photon final-state radiation in $\mathrm{Z}$ decays, forward-backward asymmetry in Drell-Yan process, and $\mathrm{W}$ muon charge asymmetry are presented.

The European Physical Society Conference on High Energy Physics

22-29 July 2015

Vienna, Austria

* Speaker.

†n behalf of the CMS Collaboration. 


\section{Introduction}

The W/Z bosons are produced with large rates at the Large Hadron Collider (LHC), and a precise measurement of the $\mathrm{W} / \mathrm{Z}$ boson production and decay properties in pp collisions allows for direct tests of the standard model (SM) predictions. Theoretical predictions are available at the next-to-next-to-leading-order (NNLO) in QCD perturbation theory [1], and the theoretical uncertainty due to parton distribution functions (PDF) dominates for many experimental observables. Therefore, a precise measurement of the $\mathrm{W} / \mathrm{Z}$ production can provide new insights into the proton structure functions. The angular quantities in the Drell-Yan process can be used to study the polarization of the $\mathrm{Z}$ boson and its coupling structure to fermions.

The measurements presented in this proceeding include the $\mathrm{Z}$ boson double differential cross section as a function of $Z$ boson transverse momentum $\left(\mathrm{q}_{T}\right)$ and rapidity $(\mathrm{y})$, the angular coefficients of $\mathrm{Z}$ decay, final state radiation (FSR) in decays of the $\mathrm{Z}$ boson, Drell-Yan forward-backward asymmetry and finally the muon charge asymmetry in W decays. All these measurements have used the full pp collision data sample collected by the CMS experiment [2] at the center-of-mass energy of $8 \mathrm{TeV}$, except that the measurement of the photon FSR uses the pp collision data at the center-of-mass energy of $7 \mathrm{TeV}$.

\section{2. $\mathrm{Z}$ boson double differential cross section at $8 \mathrm{TeV}$}

Differential measurements of $\mathrm{Z}$ boson production at the CMS have already been performed[3, 4 , 5]. In this section we present the fiducial Drell-Yan(DY) cross section, measured double differentially in terms of $\mathrm{q}_{T}$ and $\mathrm{y}$, in the muon final state from $\mathrm{Z}$ boson decays, using a dataset corresponding to an integrated luminosity of $19.7 \mathrm{fb}^{-1}$, collected by the CMS experiment at $8 \mathrm{TeV}$ proton-proton collisions [6]. With a large sample of $\mathrm{Z}$ bosons, in this measurement the high transverse momentum region, $\mathrm{q}_{T} \sim 100 \mathrm{GeV}$, of the spectrum is probed with high precision where the $\mathrm{qg} \rightarrow$ Zq sub-process dominates.

We require two well identified and isolated muons of opposite sign in the final state. The leading(and sub-leading) muon satisfies $\mathrm{p}_{T}>25(10) \mathrm{GeV}$ and $|\eta(\mu)|<2.1(2.4)$. The di-muon invariant mass satisfies $81 \mathrm{GeV}<\mathrm{M}(\mu \mu)<101 \mathrm{GeV}$. Scale factors have been applied to account for the efficiencies due to tracking, the trigger, the muon isolation and identification. The di-muon final state from $\mathrm{Z}$ boson decay is relatively clean, with backgrounds at the level of $\sim 1 \%$. The backgrounds from $t \bar{t}, \mathrm{DY} \rightarrow \tau \tau, \mathrm{WW}, \mathrm{W}+\mathrm{Jets}, t \mathrm{~W}$ and $\bar{t} \mathrm{~W}$ are estimated in a data-driven way using an independent control sample which has an opposite sign electron-muon pair in the final state. The WZ and ZZ backgrounds are estimated from simulation. The background corrected double differential inclusive $\mathrm{Z}$ spectrum is unfolded to the kinematic region as described above for the event selection criteria in 10 bins of $\mathrm{q}_{T}(\mathrm{GeV})$ : $[0,20,40,60,80,100,120,140,170,200,1000]$ and 5 bins of lyl from 0 to 2.0 with equal bin width.

In Fig. 1 the absolute differential cross section has been compared to the NNLO computation from the FEWZ calculation interfaced with the NNPDF2.3 PDFset [7]. The theoretical uncertainties include those due to PDF and QCD scale variations. As can be seen from the figure the level of agreement of this measurement with FEWZ in the $\mathrm{q}_{T}>20 \mathrm{GeV}$ region is about $10 \%$, within the experimental and theoretical uncertainties. 
In the left plot of Fig. 2 the normalized differential cross section integrated over the lyl bins is presented and compared with the prediction from FEWZ. The level of agreement between the measurement and the prediction for the range $\mathrm{q}_{T}>20 \mathrm{GeV}$ is between $6 \%-12 \%$, within the experimental and theoretical uncertainties. In the right plot of Fig. 2 the normalized differential cross section integrated over the lyl bins is compared to the predictions from the MADGRAPH [8] and POWHEG $[9,10,11,12]$. The MADGRAPH generator overestimates the data in the highest $\mathrm{q}_{T}$ bins, whereas the POWHEG generator underestimates the data up to $20 \%$ in this region.
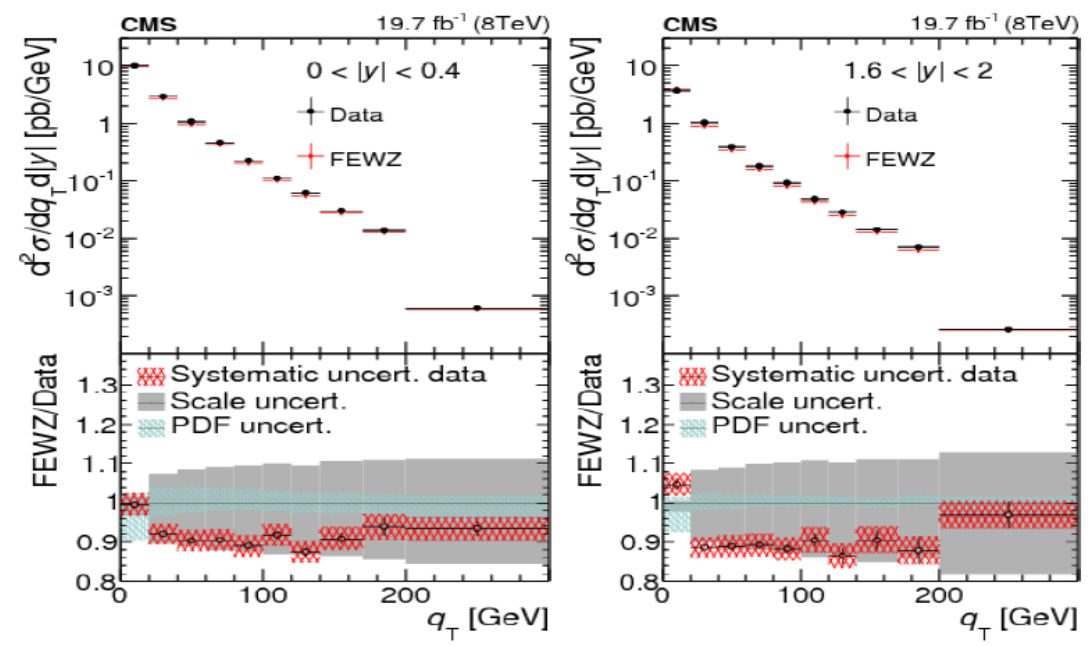

Figure 1: Absolute cross section of inclusive $\mathrm{Z}$ boson production in two bins of lyl. The theoretical prediction is from the FEWZ calculation interfaced with the NNPDF2.3 PDF set.

\section{Angular coefficients of $\mathrm{Z}$ decay at $8 \mathrm{TeV}$}

The general structure of the lepton angular distribution in the rest frame of the $\mathrm{Z}$ boson can be expressed as:

$$
\begin{aligned}
\frac{d^{2} \sigma}{d \cos \theta^{*} d \phi^{*}} \propto & {\left[\left(1+\cos ^{2} \theta^{*}\right)+A_{0} \frac{1}{2}\left(1-3 \cos ^{2} \theta^{*}\right)+A_{1} \sin \left(2 \theta^{*}\right) \cos \phi^{*}+A_{2} \frac{1}{2} \sin ^{2} \theta^{*} \cos \left(2 \phi^{*}\right)+A_{3} \sin \theta^{*} \cos \phi^{*}\right.} \\
& \left.+A_{4} \cos \theta^{*}+A_{5} \sin ^{2} \theta^{*} \sin \left(2 \phi^{*}\right)+A_{6} \sin \left(2 \theta^{*}\right) \sin \phi^{*}+A_{7} \sin \theta^{*} \sin \phi^{*}\right]
\end{aligned}
$$

In the above equation $\theta^{*}$ and $\phi^{*}$ are the polar and azimuthal angles respectively of the negatively charged lepton in the Collins-Soper frame [13]. The coefficients $A_{0}, A_{1}$, and $A_{2}$ are related to the polarization of the $\mathrm{Z}$ boson, whereas $\mathrm{A}_{3}$ and $\mathrm{A}_{4}$ are sensitive to the $\mathrm{V}$-A structure of the couplings of the muons. The only previous measurement of four of the angular coefficients was performed by the CDF Collaboration [14]. In this section we present a measurement of the angular coefficients of the $\mathrm{Z}$ boson decaying to a di-muon pair in pp collisions at $8 \mathrm{TeV}$ with an integrated luminosity of $19.7 \mathrm{fb}^{-1}$ [15].

The event selection criteria, the correction factors applied and the background estimation technique are the same as that for the measurement in Section 2. The coefficients $\mathrm{A}_{0}-\mathrm{A}_{4}$ are extracted 

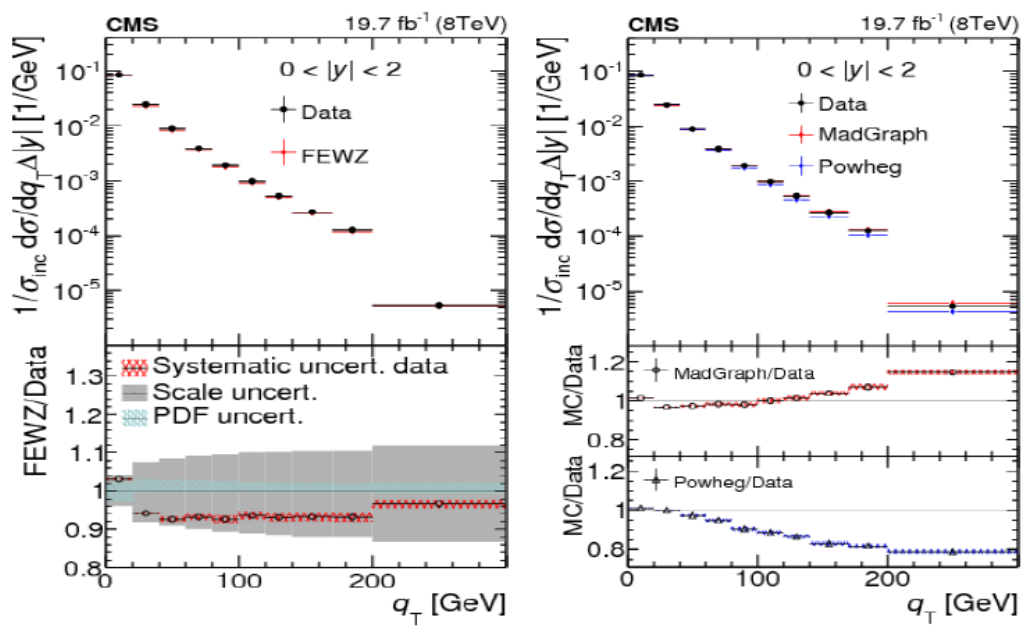

Figure 2: (Left) Cross section of inclusive $\mathrm{Z}$ boson production normalized to the total inclusive cross section. The theoretical prediction is from the FEWZ calculation interfaced with the NNPDF2.3 PDF set.

(Right) Normalized Z-boson cross section, compared to predictions of simulation from MADGRAPH (red symbols) and POWHEG (blue symbols). MADGRAPH uses the CTEQ6L1 PDF set [16] and POWHEG the CT10(NLO) PDF set [17].

by fitting the $\cos \theta^{*}$ and $\phi^{*}$ distributions from data, using simulated signal and background templates. A binned maximum likelihood method with Poissonian uncertainties is used for the fits. The measurements are carried out in 8 bins of $\mathrm{q}_{T}(\mathrm{GeV}):[0,10,20,35,55,80,120,200,1000]$ and 2 bins of |y|: $[0,1.0,2.1]$. The results obtained from these fits are compared to the predictions from MADGRAPH, POWHEG and FEWZ for one of the rapidity bins in Fig. 3. The PDF sets used in these predictions are CTEQ6L, CT10(NLO) and CT10(NNLO), respectively. The MADGRAPH predictions for $\mathrm{A}_{4}$ are systematically higher than those from POWHEG and FEWZ as MADGRAPH uses a weak mixing angle calculated without taking into account the radiative corrections. The measured $\mathrm{A}_{0}$ and $\mathrm{A}_{2}$ coefficients show a better agreement with the prediction from MADGRAPH than with those of POWHEG and FEWZ, especially at high $\mathrm{q}_{T}$. A violation of the Lam-Tung relation $\left(\mathrm{A}_{0}=\right.$ $\mathrm{A}_{2}$ ) is observed, which is anticipated by QCD calculations beyond the leading order. These results will play an important role in future high-precision measurements, such as the measurement of the mass of the $\mathrm{W}$ boson and of the electroweak mixing angle.

\section{Final state radiation in decays of $\mathrm{Z}$ boson at $7 \mathrm{TeV}$}

In this measurement the differential cross section for photon production in $\mathrm{Z} \rightarrow \mu^{+} \mu^{-} \gamma$ decays is carried out using data from pp collisions at $7 \mathrm{TeV}$ collected by the CMS detector corresponding to an integrated luminosity of $4.7 \mathrm{fb}^{-1}$ [18]. Such radiative decays of the $\mathrm{Z}$ boson were noted in the very first $Z$ boson publications of UA1 and UA2 $[19,20]$. The differential cross sections $\frac{d \sigma}{d E_{T}}$, where $\mathrm{E}_{T}$ is the transverse energy of the photon, and $\frac{d \sigma}{d \Delta R_{\mu \gamma}}, \Delta R_{\mu \gamma}=\sqrt{\left(\eta_{\mu}-\eta_{\gamma}\right)^{2}+\left(\phi_{\mu}-\phi_{\gamma}\right)^{2}}$ with $\phi$ the azimuthal angle (in radians) around the beam axis and $\eta$ the rapidity, are presented. The goal of this analysis is to establish the quality of the modeling of FSR photons emitted in Z boson decays 


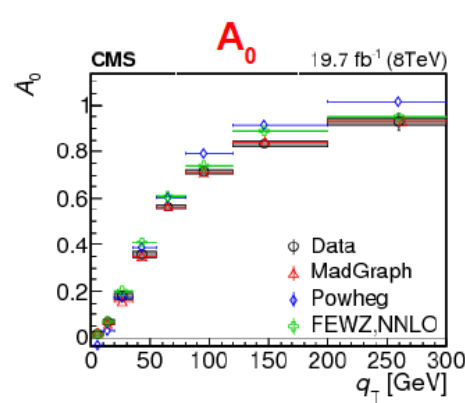

$\mathrm{A}_{3}$

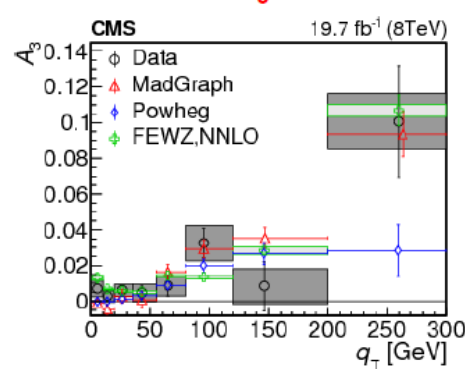

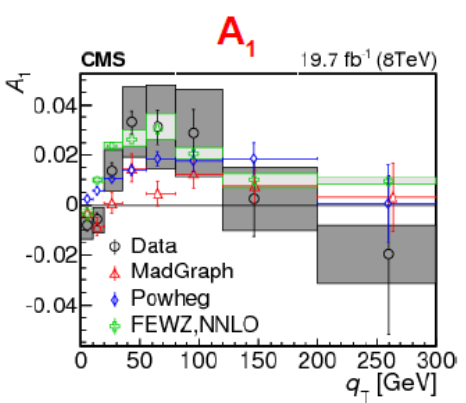

$\mathrm{A}_{4}$

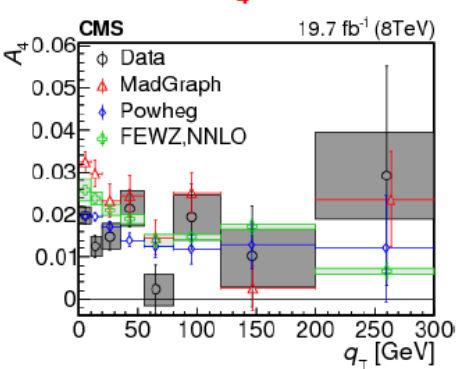

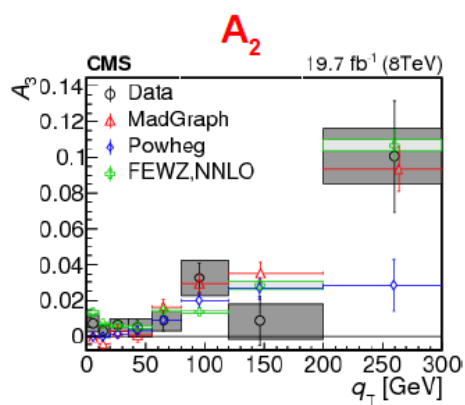

$A_{0}=A_{2}$

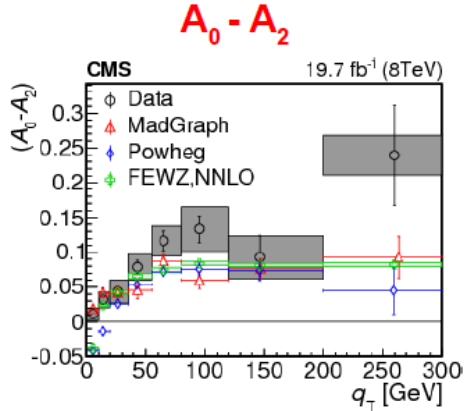

Figure 3: Comparison of the 5 angular coefficients $A_{i}$ and $A_{0}-A_{2}$ for $|y|<1$. The circles show the measured results. The vertical bars represent the statistical uncertainties and the boxes the systematic uncertainties of the measurement. The triangles show the predictions from MADGRAPH, the diamonds from POWHEG, and the crosses from FEWZ at NNLO. The boxes at the FEWZ values indicate the PDF uncertainties.

over a wide kinematic and angular range. This result can support future measurements of the $\mathrm{W}$ mass, the study of $Z+\gamma$ production, and searches for new particles in final states with photons. This is the first such measurement performed at the LHC experiments.

The selected events must have two well identified and isolated muons in the final state. The leading(sub-leading) muon satisfies $\mathrm{p}_{T}>31(9) \mathrm{GeV}$ and $|\eta(\mu)|<2.4(2.4)$. The di-muon invariant mass satisfies $30 \mathrm{GeV}<\mathrm{M}(\mu \mu)<87 \mathrm{GeV}$. Backgrounds to this measurement come mainly from "non-prompt" photons which may be genuine photons produced in the decays of light mesons (such as $\pi^{0}$ and $\eta$ ) and photons from pileup. This is estimated from DY events suitably accounting for the differences in data and simulation. Other background processes that contribute to a much lesser extent are $t \bar{t}, \mathrm{DY} \rightarrow \tau \tau$ and di-boson production. These are estimated from simulation. The contribution from $\mathrm{W}+$ jets and QCD multi-jet production has been estimated using events with same sign muons. The background subtracted distribution for $\mathrm{E}_{T}$ and $\Delta R_{\mu \gamma}$ are unfolded to the kinematic and fiducial region as required by the event selection criteria described earlier.

Fig. 4 shows the measured differential cross sections $\frac{d \sigma}{d E_{T}}$ and $\frac{d \sigma}{d \Delta R_{\mu \gamma}}$ compared with the theoretical prediction from POWHEG and PYTHIA 6, where PYTHIA is used to simulate parton showers and final-state photons. The CT10 PDF set is used for this prediction. The theoretical uncertainties include those due to PDF and QCD scale variations. The agreement with data is is better than 5\%.

\section{Drell-Yan forward-backward asymmetry at $8 \mathrm{TeV}$}

The presence of the vector and axial-vector couplings of the electroweak bosons to fermions 

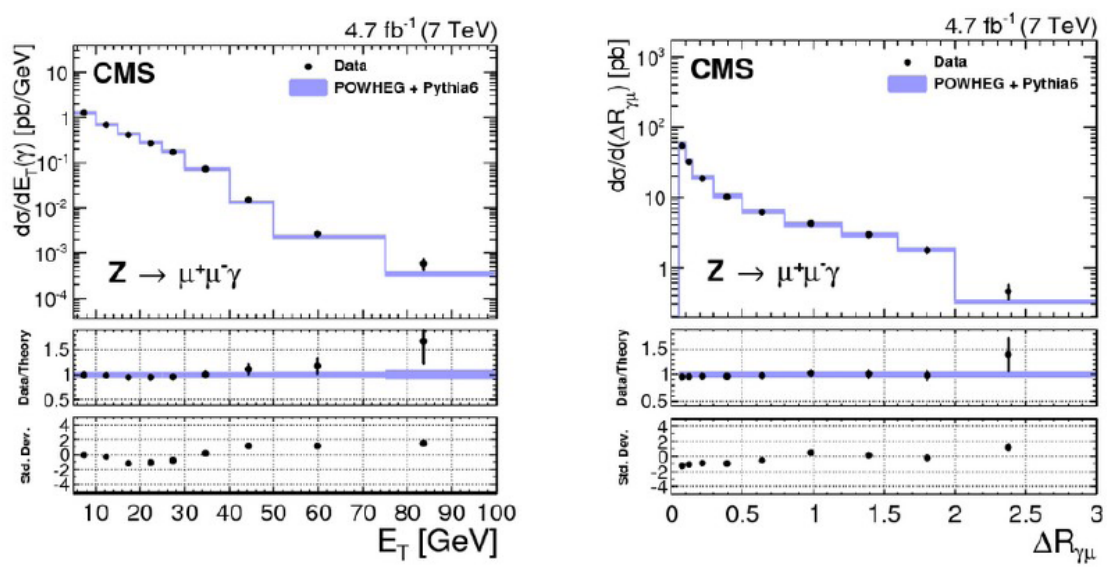

Figure 4: Measured differential cross sections $\mathrm{d} \sigma / \mathrm{dE} \mathrm{E}_{T}$ (left) and $\mathrm{d} \sigma / \mathrm{d} \Delta \mathrm{R}_{\mu \gamma}$ (right). In the upper panels, the dots with error bars represent the data, and the shaded bands represent the POWHEG+PYTHIA calculation including theoretical uncertainties. The lower panels show the standard deviations of the measurements with respect to the calculation.

in the standard-model (SM) process $\mathrm{q} \bar{q} \rightarrow \mathrm{Z} / \gamma^{*} \rightarrow 1^{+} 1^{-}$results in a forward-backward asymmetry in the number of Drell-Yan lepton pairs. For a given dilepton mass, the differential cross section at the parton level at leading order (LO) can be expressed as:

$$
\frac{d \sigma}{d \cos \theta}=A\left(1+\cos ^{2} \theta\right)+B \cos \theta
$$

where $\theta$ represents the emission angle of the negatively-charged lepton relative to the quark momentum in the center-of-mass frame of the dilepton system, while A and B are parameters that depend on the weak isospin and the charge of the incoming fermions. The forward and backward asymmetry parameter, $\mathrm{A}_{F B}$, is then defined as:

$$
A_{F B}=\frac{\sigma_{F}-\sigma_{B}}{\sigma_{F}+\sigma_{B}}
$$

where $\sigma_{F}\left(\sigma_{B}\right)$ are the total cross section for the forward (backward) events, defined by $\cos \theta>0$ $(<0)$. The $\mathrm{A}_{F B}$ parameter depends on the dilepton mass, quark flavor and the electroweak mixing angle $\theta_{W}$. Near the $\mathrm{Z}$ peak the $\mathrm{A}_{F B}$ parameter is close to zero because of the small value of the lepton vector coupling. To reduce the uncertainties due to the transverse momentum of the incoming quarks, the measurement is carried out in the Collins-Soper frame. The $\mathrm{A}_{F B}$ parameter was previously measured at CMS using a data sample collected in $7 \mathrm{TeV}$ pp collisions [21]. In this section we present a measurement of the forward-backward asymmetry $\mathrm{A}_{F B}$ of oppositely charged lepton pairs produced via an intermediate $\mathrm{Z} / \gamma^{*}$. The dataset used corresponds to an integrated luminosity of $19.7 \mathrm{fb}^{-1}$ in pp collisions at $8 \mathrm{TeV}$ collected with the CMS detector. The measurement is carried out both in the dimuon and dielectron final states [22].

The kinematic criteria required in this measurement is $\mathrm{p}_{T}>20 \mathrm{GeV}$ and $|\eta|<2.4$ for both the muons in the dimuon final state. There are two categories of electrons considered in this measurement. For one case both electrons satisfy $\mathrm{p}_{T}>20 \mathrm{GeV}$ and $|\eta|<2.4$. In order to pick up events 
in the more forward region a second set of event selection criteria is used only in the dielectron final state that requires the leading electron to satisfy $\mathrm{p}_{T}>30 \mathrm{GeV}$ and $|\eta|<2.4$ and the sub-leading electron must have $\mathrm{p}_{T}>20 \mathrm{GeV}$ and $3.0<|\eta|<5.0$. The backgrounds to this channel are at the $1 \%$ level and are estimated using both simulation as well as data-driven techniques. The measurement is carried out as a function of the di-lepton invariant mass in 5 absolute rapidity bins from $0-5$. The distributions are corrected for the effects of detector resolution using an unfolding technique. Results in the two channels for the dilepton rapidity range, $|y|<2.4$ are combined assuming the systematics are uncorrelated between the electron and muon channels. Fig. 5 shows the results of the measurement. The distribution in the range $2.4<|y|<5.0$ is from the electron channel only whereas the rest are from a combination of the two channels. The theoretical prediction is from the POWHEG generator with parton showering and hadronization done using the PYTHIA6 event generator. The CT10 PDF sets have been used for the prediction with the theoretical uncertainty due to PDF and QED final state radiation effects considered. The dominant systematic uncertainty in the measurement is due to that from the backfround estimation. The measured results are in good agreement with the theoretical prediction.
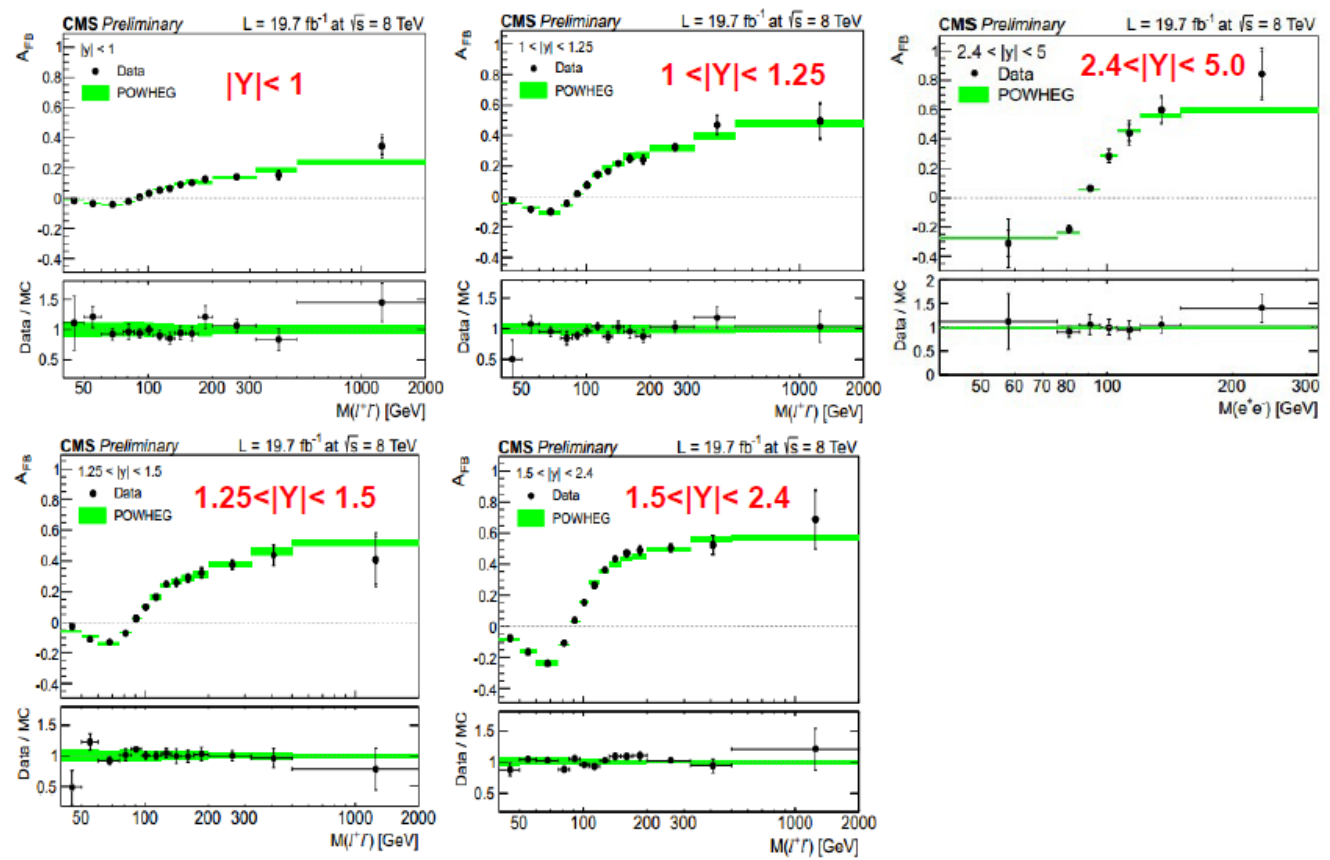

Figure 5: Combined $A_{F B}$ distributions from the dielectron and dimuon channels for $|y|<2.4$, and only from the dielectron channel for $|y|>2.4$. The inner thick vertical bars correspond to the statistical uncertainty and the outer thin vertical bars show the total uncertainties. The corresponding POWHEG predictions are shown as the green bands with the total uncertainty (stat. $\oplus$ PDF).

\section{Muon charge asymmetry in $\mathrm{W}$ decays at $8 \mathrm{TeV}$}

Due to the presence of two valence u-quarks in the proton there is an overall excess of $\mathrm{W}^{+}$over $\mathrm{W}^{-}$. The asymmetry is measured as a function of the pseudo-rapidity $\eta$ of the muon in the CMS laboratory frame, and is defined as follows: 


$$
A(\eta)=\frac{\frac{d \sigma}{d \eta}\left(W^{+} \rightarrow \mu^{+} v\right)-\frac{d \sigma}{d \eta}\left(W^{-} \rightarrow \mu^{-} v\right)}{\frac{d \sigma}{d \eta}\left(W^{+} \rightarrow \mu^{+} v\right)+\frac{d \sigma}{d \eta}\left(W^{-} \rightarrow \mu^{-} v\right)}
$$

At LHC the lepton charge asymmetries have been measured by the CMS, ATLAS and LHCb experiments as can be found in $[23,24,25]$. In this section the measurement of the muon charge asymmetry in inclusive $\mathrm{pp} \rightarrow \mathrm{W}^{ \pm}+\mathrm{X} \rightarrow \mu^{ \pm} v+\mathrm{X}$ events is presented [26]. The dataset used corresponds to an integrated luminosity of $18.8 \mathrm{fb}^{-1}$ in pp collisions at $8 \mathrm{TeV}$ collected with the CMS detector.

The events are selected requiring one well identified and isolated muon in the final state. This muon must satisfy $\mathrm{p}_{T}>25 \mathrm{GeV}$ and $|\eta|<2$.4. In order to veto events that may arise from DY production, events with a sub-leading muon having $\mathrm{p}_{T}>15 \mathrm{GeV}$ are rejected. The backgrounds to this measurement arise out of QCD multijets, DY $\rightarrow \mu^{+} \mu^{-}$production and to a lesser extent from $\mathrm{W} \rightarrow \tau \nu$ and $\mathrm{DY} \rightarrow \tau \tau$ events. The measurement is carried out in eleven bins of the $|\eta|$ of the muon varying from $0.0-2.4$. In each $|\eta|$ bin templates of the missing transverse energy(MET) are constructed from simulation which are then fitted to the data. The fits are performed simultaneously to extract the number of $\mathrm{W}^{+}$and $\mathrm{W}^{-}$events. The total uncertainty of this measurement is $\sim$ $0.2 \%-0.26 \%$ dominated by the uncertainty due to the QCD background estimation. In Fig. 6 the measured charge asymmetry has been compared to the prediction from the FEWZ calculation interfaced with the CT10, NNPDF3.0, HERAPDF1.5 and MMHT2014 PDF sets. The measured charge asymmetries are well described by all considered PDF models within their corresponding uncertainties. This measurement has been used to constrain the PDFs of the valence $u$ and $d$ quarks and a clear improvement in precision has been observed over the entire range of Bjorken $\mathrm{x}$.

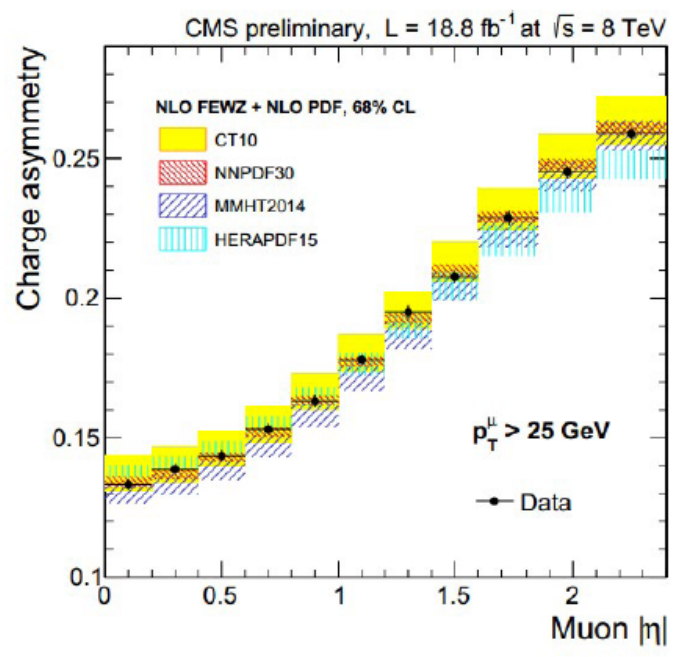

Figure 6: Comparison of the measured muon charge asymmetries to NLO predictions calculated using the FEWZ calculation interfaced with four different PDF sets. The vertical error bars on data points include both statistical and systematic uncertainties. The PDF uncertainty for each PDF set is shown by the shaded/hatched band and corresponds to $68 \% \mathrm{CL}$. 


\section{Summary}

In this proceeding we have presented several measurements with W/Z events in pp collisions at $\sqrt{s}$ of both 7 and $8 \mathrm{TeV}$. The results are compared with the standard model predictions and in general good agreements are achieved. The uncertainties in these measurements are well understood and are mostly below that of the corresponding theoretical predictions. Hence more precise theoretical calculations are needed. The measured $\mathrm{W}$ muon charge asymmetry can be used to constrain PDF uncertainties in future global PDF analyses.

\section{References}

[1] R. Gavin et al., "FEWZ 2.0: A code for hadronic Z production at next-to-next-to-leading order", Comput. Phys. Commun. 182 (2011) 2388 [arXiv:1011.3540].

[2] CMS Collaboration, "The CMS experiment at the CERN LHC", JINST 3:S08004,2008.

[3] CMS Collaboration, "Measurement of the rapidity and transverse momentum distributions of $Z$ bosons in pp collisions at $\sqrt{s}=7$ TeV", Phys. Rev. D 85 (2012) 032002 [arXiv:1110.4973].

[4] CMS Collaboration, "Measurement of the differential and double-differential Drell-Yan cross sections in proton-proton collisions at $\sqrt{s}=7$ TeV", JHEP 12 (2013) 030 [arXiv:1310.7291].

[5] CMS Collaboration, "Measurements of differential and double-differential Drell-Yan cross sections in proton-proton collisions at $\sqrt{s}=8$ TeV", Eur. Phys. J C75 (2015), no. 4, 147 [arXiv:1412.1115].

[6] CMS Collaboration, "Measurement of the Z boson differential cross section in transverse momentum and rapidity in proton-proton collisions at 8 TeV", Phys. Lett. B 749 (2015) 187-209 [arXiv:1504.03511].

[7] R. D. Ball et al., "A first unbiased global NLO determination of parton distributions and their uncertainties", Nucl. Phys. B 838 (2010) 136-206 [arXiv:1002.4407].

[8] J. Alwall et al., "The automated computation of tree-level and next-to-leading order differential cross sections, and their matching to parton shower simulations", JHEP 07 (2014) 079 [arXiv:1405.0301].

[9] P. Nason, "A new method for combining NLO QCD with shower Monte Carlo algorithms", JHEP 11 (2004) 040 [arXiv:hep-ph/0409146].

[10] S. Frixione et al., "Matching NLO QCD Computations with Parton Shower Simulations: the POWHEG method", JHEP 11 (2007) 070 [arXiv:0709.2092].

[11] S. Alioli et al., "NLO Vector-Boson Production Matched with Shower in POWHEG", JHEP 07 (2008) 060 [arXiv:0805.4802].

[12] S. Alioli et al., "A general framework for implementing NLO calculations in shower Monte Carlo programs: the POWHEG BOX", JHEP 06 (2010) 043 [arXiv:1002.2581].

[13] J. Collins and D. Soper, "Angular Distribution of Dileptons in High-Energy Hadron Collisions", Phys. Rev. D 1616 (1977) 2219.

[14] CDF Collaboration, "First Measurement of the Angular Coefficients of Drell-Yan $e^{+} e^{-}$pairs in the Z Mass region from p $\bar{p}$ collisions at $\sqrt{s}=1.96$ TeV", Phys. Rev. Lett. 106 (2011) 241801 [arXiv:1103.5699]. 
[15] CMS Collaboration, "Angular coefficients of $Z$ bosons produced in pp collisions at $\sqrt{s}=8$ TeV and decaying to $\mu^{+} \mu^{-}$as a function of transverse momentum and rapidity", Phys. Lett. B 750 (2015) 154-175 [arXiv:1504.03512].

[16] J. Pumplin et al., "New generation of parton distributions with uncertainties from global QCD analysis", JHEP 07012 [arXiv:hep-ph/0201195].

[17] H.-L. Lai et al., "New parton distributions for collider physics", Phys. Rev. D 82 (2010) 074024 [arXiv:1007.2241].

[18] CMS Collaboration, "Study of Final-State Radiation in Decays of Z Bosons produced in pp Collisions at 7 TeV", Phys. Rev. D 91 (2015) 092012 [arXiv:1502.07940].

[19] C. Albajar et al. (UA1), "Studies of intermediate vector boson production and decay in UA1 at the CERN proton-antiproton collider", Z. Phys. C 44, 15 (1989).

[20] P. Bagnaia et al. (UA2), "Evidence for $Z^{0} \Rightarrow e^{+} e^{-}$at the CERN $\bar{p} p$ collider", Phys. Lett 129B, 130 (1983).

[21] CMS Collaboration, "Forward backward asymmetry of Drell Yan lepton pairs in pp collisions at $\sqrt{s}=$ 7 TeV", Phys. Lett. B 718 (2013) 752.

[22] CMS Collaboration, "Forward-backward asymmetry of Drell-Yan lepton pairs in pp collisions at 8 $T e V "$, CMS Physics Analysis Summary CMS-PAS-SMP-14-004, 2015, https://cds.cern.ch/record/2011635.

[23] ATLAS Collaboration, "Measurement of the inclusive $W^{ \pm}$and Z/gamma cross sections in the electron and muon decay channels in pp collisions at $\sqrt{s}=7$ TeV with the ATLAS detector", Phys. Rev. D 85 (2012) 072004 [arXiv:1109.5141].

[24] CMS Collaboration, "Measurement of the lepton charge asymmetry in inclusive W production in pp collisions at $\sqrt{s}=7$ TeV", JHEP 04 (2011) 050 [arXiv:1103.3470].

[25] LHCb Collaboration, "Inclusive $W$ and Z production in the forward region at $\sqrt{s}=7$ TeV", JHEP 06 (2012) 058 [arXiv:1204.1620].

[26] CMS Collaboration, "Measurement of the muon charge asymmetry in inclusive pp to WX production at $\sqrt{s}=8 T e V^{\prime \prime}$, CMS Physics Analysis Summary CMS-PAS-SMP-14-022, 2015, https://cds.cern.ch/record/2013329. 